\title{
Associations of Serum Total Bilirubin with Survival Outcomes in Patients with Cancer Cachexia: A Retrospective, Multicenter Cohort Study
}

\section{Xiang-Rui Li}

Capital Medical University Affiliated Beijing Shijitan Hospital

Qi Zhang

Capital Medical University Affiliated Beijing Shijitan Hospital

\section{Kang-Ping Zhang}

Capital Medical University Affiliated Beijing Shijitan Hospital

\section{Xi Zhang}

Capital Medical University Affiliated Beijing Shijitan Hospital

\section{Guo-Tian Ruan}

Capital Medical University Affiliated Beijing Shijitan Hospital

\section{Meng-Meng Song}

Capital Medical University Affiliated Beijing Shijitan Hospital

\section{Yi-Zhong Ge}

Yuying Children's Hospital of Wenzhou Medical College: Wenzhou Medical University Second Affiliated Hospital

\section{Xiao-Wei Zhang}

Capital Medical University Affiliated Beijing Shijitan Hospital

\section{Chun-Hua Song}

Zhengzhou University

Han ping Shi ( $\square$ shihp@ccmu.edu.cn )

Capital Medical University Affiliated Beijing Shijitan Hospital https://orcid.org/0000-0003-4514-8693

\section{Research}

Keywords: Total bilirubin, Liver function, Cancer cachexia, Prognostic

Posted Date: July 26th, 2021

DOl: https://doi.org/10.21203/rs.3.rs-675549/v1

License: (c) (1) This work is licensed under a Creative Commons Attribution 4.0 International License. Read Full License 


\section{Abstract}

Background: Cancer cachexia is a systemic paraneoplastic phenomenon involving multiple organs, including the liver. Total bilirubin (TBIL) is an easily obtained blood biomarker that reflects liver homeostasis. This work evaluated the prognostic value of serum TBIL in patients with cancer cachexia.

Methods: This study included 2,282 patients from a multicenter research database who were diagnosed with cancer cachexia between June 2012 and December 2019. The hazard ratio (HR) for all-cause mortality was analyzed using Cox proportional hazards regression models. The association of serum TBIL with all-cause mortality was modeled with restricted cubic splines. The optimal cutoff value for TBIL was calculated with maximally selected rank statistics.

Results: Among the participants, there were 1,327 (58.2\%) males and 955 (41.8\%) females. The mean patient age was $60.4 \pm 1.5$ years. The 12-month all-cause mortality rate for patients with cancer cachexia was $29.5 \%$ (95\% Cl: $27.6 \%-31.3 \%)$, resulting in a rate of 209.58 events per 1000 patient-years. An inverted L-shaped association between TBIL and all-cause mortality was observed. The cutoff point for TBIL for the prediction of the time to mortality was $<21.7 \mu \mathrm{mol} / \mathrm{L}$. A high TBIL level but not the direct bilirubin (DBIL) or indirect bilirubin (IBIL) level was identified as an independent prognostic factor (HR, 1.60, 95\% $\mathrm{Cl}, 1.32-1.93)$. For patients with digestive system tumors, a high serum TBIL level ( $\geq 21.7 \mu \mathrm{mol} / \mathrm{L})$ was significantly associated with mortality. Conclusion: High TBIL levels are associated with increased allcause mortality in patients and might be a promising prognostic indicator in patients with cancer cachexia.

\section{Introduction}

Cancer cachexia is a systemic syndrome involving progressive body weight and muscle reductions, systemic inflammation as well as adipose tissue wasting that seriously affects patient quality of life and shortens the lifespan ${ }^{1,2}$. Cachexia affects $70 \%$ of cancer patients and accounts for at least $22 \%$ cancerrelated death cases ${ }^{3}$.Currently, there is no available standard treatment to fully reverse cancer cachexia ${ }^{4}$.

Cachexia Experts have reached an agreement on the definition and classification of cancer cachexia ${ }^{1}$. Cancer cachexia was divided into three stages: precachexia, cachexia, and refractory cachexia. Based on this model, Blum et al improved the classification system by further developing a four-group model that improve the accuracy of ability of discrimination ${ }^{5}$. Importantly, high heterogeneity in the prognosis of patients with cancer cachexia has been observed ${ }^{6}$. However, there is no effective method of assessing the prognosis of patients with different stages of cachexia according to these classification systems. Notably, prognostic biomarkers help not only identify patients with poor clinical outcomes but also stratify patients based on the optimal treatment or eligibility for clinical trials. Therefore, there exists a significant demand for easily determinable and readily available prognostic biomarkers to guide the assessment of life expectancy. 
Cancer cachexia can affect and/or be influenced by many organs, such as the liver ${ }^{7}$. It has long been known that the liver is a major contributor to cancer cachexia due to the presence of systemic inflammation and hepatic metabolism alterations ${ }^{8,9}$. Bilirubin (BLB), as the final product of heme degradation, is essential to reflect liver function. For many years, BLB has been regarded as an inauspicious sign of liver dysfunction with very limited physiological benefits ${ }^{10}$. Temme et al found high serum total bilirubin (TBIL) levels, albeit within the normal range, was associated with reduced cancer mortality, especially nonlung cancer mortality $(P$ for trend $<0.02)$ among 5,460 men and 4,843 women during a 10 -year follow-up ${ }^{11}$. Consistent with this finding, recent studies showed that mildly elevated TBIL levels may be associated with reduced prevalences of cardiovascular diseases (CVDs), cancer, and metabolic syndrome ${ }^{12,13}$. However, the highest serum levels of TBIL were found in patients with cardiac cachexia, and they were correlated with reduction in systolic right ventricle (RV) function and increased right atrial pressure ${ }^{14}$. Cancer cachexia is complex, with a multifactorial etiology, and the association between serum TBIL levels and mortality in patients with cancer cachexia remains unclear. Blood is easily accessible and obtaining a blood sample is a minimally invasive procedure, which makes blood biomarkers appealing for use in clinical practice. TBIL has great potential for the prediction of individual clinical outcomes and the stratification of patients into different prognostic subgroups.

Herein, we established the cutoff point for TBIL for the prediction of all-cause mortality in Chinese cancer patients with cachexia. In addition, a summary analysis of the clinical parameters of the patients was conducted to evaluate the potential associations of those parameters with the serum TBIL level. The aim of this study was to evaluate the potential prognostic value of the serum TBIL level in patients with cancer cachexia.

\section{Patient And Methods}

\subsection{Study Participants}

Briefly, this retrospective cohort study included 12,792 patients age 22 to 94 years who were diagnosed with malignant cancer based on pathology and were enrolled at more than 40 clinical centers throughout China from June 1, 2012, through December 31, 2019. Patients with multiple hospitalizations were regarded as one case. All patients provided written informed consent. No other special exclusion criteria were imposed except evidence of hemolytic disease and refusal to participate in the study. Patients with insufficient data concerning body mass index (BMI), blood test results, European Organization for Research and Treatment of Cancer Quality of Life Questionnaire (EORTC QLQ-C30 Version 3.0) responses, and survival duration were excluded, leaving 2,282 patients with cancer cachexia for inclusion in the current analysis (Fig. 1). The average age of recipients was $60.4 \pm 1.5$ years with $41.8 \%$ (955/2282) of them were female. The study was approved by the institutional review board and complied with current ethics guidelines.

\subsection{Data Collection and Definitions}


Diagnoses of malignant cancers were based on histological evidence after operation or needle biopsy. Baseline information, including age, sex, BMI, comorbidities (diabetes mellitus, hypertension, and hepatobiliary disease), alcohol consumption, alcohol consumption, tea consumption, cancer types, TNM stage, treatment method, mid-arm circumference (MAC), hand-grip strength (HGS), anorexia, sarcopenia, patient-generated subjective global assessment (PG-SGA), EORTC QLQ-C30 and nutrition interventions, was recorded at the time of diagnosis. TNM staging was conducted using the 8th Union for International Cancer Control TNM staging system. All biochemical indicators, such as serum albumin concentration, TBIL, direct bilirubin (DBIL), indirect bilirubin (IBIL), alanine aminotransferase (AST), aspartate aminotransferase (ALT), white blood cell count, neutrophil count, lymphocyte count, red blood cell (RBC) count, and platelet count, were evaluated before treatment (surgery, chemotherapy, radiotherapy, and other treatment) at the baseline visit. The EORTC QLQ-C30 includes 30 items that are summarized in a global health status subscale (dyspnea, insomnia, appetite loss, constipation, diarrhea, and financial difficulties), functional subscales (physical, role, social, emotional, and cognitive), and symptom subscales (fatigue, pain, and nausea and vomiting) ${ }^{15}$.

\subsection{Definition of Cachexia}

The international consensus criterion for cancer cachexia was weight loss more than $5 \%$ over the past 6 months (in the absence of simple starvation) or weight loss greater than $2 \%$ in individuals already underweight according to current $\mathrm{BMI}\left(\mathrm{BMI}<20 \mathrm{~kg} / \mathrm{m}^{2}\right.$ ) or skeletal muscle mass (sarcopenia). Skeletal muscle depletion was assessed based on mid-upper-arm muscle area according to anthropometry (men $<32 \mathrm{~cm}^{2}$, women $\left.<18 \mathrm{~cm}^{2}\right)^{1}$. Cancer cachexia was diagnosed when any one of the criteria was satisfied.

\subsection{Statistical Analyses}

Overall survival (OS) was defined as the interval between the first assessment in the clinic until the date of death, or end of follow-up (September 31, 2019), or time of last contact, whichever came first. The Kolmogorov-Smirnov test was used to test the normality of continuous data. Normally distributed data Data are presented as the means \pm SD or as the medians and interquartile ranges (IQRs) for data with skewed distribution. Serum TBIL was log-transformed before analysis. A Cox proportional hazards model was used for univariate and multivariate OS analyses. Hazard ratios (HRs) and their $95 \%$ confidence intervals (Cls) were reported. Correlations between TBIL and nutritional parameters were explored using pairwise Spearman's rank correlation coefficients. A possible nonlinear relationship between TBIL and HR was analyzed by restricted cubic spline regression. TBIL was categorized based on an optimal cutoff point of $21.7 \mu \mathrm{mol} / \mathrm{L}$ obtained by maximizing the sensitivity and specificity. Survival endpoints were assessed by Kaplan-Meier survival curves and compared by the log-rank test. Separate Cox models were applied to each subgroup in stratified covariate subgroup analyses. The Wald test were used to tested for trends across the median value for each quartile. Interaction tests were conducted with cross-classified multilevel interaction terms in the multivariable models. Kaplan-Meier survival curves are shown for the subgroups with significant $(P$ for interaction $<0.1)$ interaction terms. All the statistical analysis was performed using R software (version 4.0.2). 


\section{Results}

\subsection{Characteristics of Serum TBIL in Patients with Cancer Cachexia}

A total of 2,282 patients of cancer-related cachexia were diagnosed among 12,279 participants. By the end of the study, (December 31, 2019), 1,245 (5\%) death cases were identified. The serum TBIL levels were higher in liver cancer patients and lower in patients with nasopharyngeal cancer and colorectal cancer (CRC) (Fig. 2). No association between the serum TBIL concentration and tumor stage was observed (Supplementary Fig. 1). Further potential associations between serum TBIL levels and covariates in patients with cancer cachexia are presented in Supplementary Fig. 2. The serum TBIL levels were positively correlated with AST and ALT levels. Weak correlations were found between serum TBIL levels and other clinical indexes (age, BMI, albumin, platelet count, RBC count, HGS, Karnofsky Performance Status (KPS) score, EORTC QLQ-C30 score, and PG-SGA score).

\subsection{Association of Serum TBIL with Overall Survival}

The results of the univariate and multivariate Cox regression analyses of the associations of serum TBIL levels with OS in cancer cachexia patients are shown in Supplementary Table 1. Most baseline characteristics were associated with an increased risk of mortality in the univariate analyses. Age, tumor type, TNM stage, albumin, TBIL, neutrophil count, AST, ALT, RBC count, platelet count, HGS, sarcopenia, and EORTC QLQ-C30 score were independent prognostic factors in multivariate analysis. However, DBIL and IBIL were not independent prognostic markers. Restricted cubic spline models revealed that the risk of mortality was positive correlated with the serum TBIL level(Fig. 3). In addition, TBIL was associated with a poor prognosis in patients with cancer cachexia after adjustment for sex, age, tumor type, TNM stage, alcohol intake, albumin, neutrophil count, AST, ALT, RBC count, platelet count, HGS, sarcopenia, and EORTC QLQ-C30 score (Table 1). Each increase of one standard deviation of serum Tbil was significantly associated with a $13 \%$ increase in the risk of mortality in Model b $(\mathrm{HR}, 1.13 ; 95 \% \mathrm{Cl}, 1.08-1.18 ; P<0.001)$. When the serum TBIL level was divided into tertiles, tertiles 2 (HR, 1.16;95\% $\mathrm{Cl}, 1.04-1.39)$ and $3(\mathrm{HR}$, $1.32 ; 95 \% \mathrm{Cl}, 1.14-1.54)$ were associated with a higher risk of mortality $(P$ for trend $<0.001)$. 
Table 1

The association between TBIL and hazard ratio of cachexia patients

\begin{tabular}{|c|c|c|c|c|}
\hline TBIL & Model a & & Model b & \\
\hline & $\mathrm{HR} 95 \% \mathrm{Cl}$ & $P$-value & $\mathrm{HR} 95 \% \mathrm{Cl}$ & $P$-value \\
\hline As continuous (per SD) & $1.17(1.13,1.22)$ & $<0.001$ & $1.13(1.08,1.18)$ & $<0.001$ \\
\hline \multicolumn{5}{|l|}{ By TBIL, $\mu \mathrm{mol} / \mathrm{L}$ cut-off } \\
\hline Low $(<21.7)$ & ref & & ref & \\
\hline High $(\geq 21.7)$ & $1.93(1.62,2.30)$ & $<0.001$ & $1.60(1.32,1.93)$ & $<0.001$ \\
\hline \multicolumn{5}{|l|}{ Tertile, $\mu \mathrm{mol} / \mathrm{L}$} \\
\hline Q1 (<9.1) & ref & & ref & \\
\hline Q2 (9.1-13.5) & $1.16(1.04,1.39)$ & 0.040 & $1.26(1.08,1.48)$ & 0.004 \\
\hline Q3 ( $\geq 13.5)$ & $1.32(1.14,1.54)$ & $<0.001$ & $1.45(1.24,1.70)$ & $<0.001$ \\
\hline$p$ for trend & & $<0.001$ & & $<0.001$ \\
\hline \multicolumn{5}{|c|}{ Model a: adjusted by gender, age, BMI, and TNM stage; } \\
\hline
\end{tabular}

\subsection{Demographics and Disease Characteristics Dichotomized by TBIL in Patients with Cancer Cachexia}

Patients was further stratified according to the cutoff point of $21.7 \mu \mathrm{mol} / \mathrm{L}$ that was the best for the prediction of OS (Supplementary Fig. 3A). Kaplan-Meier curves revealed that patients with high TBIL levels had poorer OS (Supplementary Fig. 3B). Table 2 presents the characteristics of 234 patients with cancer cachexia stratified by high and non-high prediagnosis levels of TBIL. Patients with high TBIL levels tended to be older and with higher percent of male, anorexia, hepatobiliary disease, and receive radiotherapy and chemotherapy. Higher TBIL accompanied by other biochemical criteria: higher neutrophil count, AST, ALT, and higher DBIL level, lower albumin, lymphocyte count, RBC count, and lower platelet count. Furthermore, a higher TBIL level was associated with a higher PG-SGA score, and EORTC QLQ-C30 score (Supplementary Table 2), lower KPS score and nutrition support. Although the distribution of TBIL levels differed among cancer populations, TBIL levels were significantly associated with the OS of patients with digestive system tumors, especially those with CRC. (data not shown) (Fig. 4). 
Table 2

Baseline characteristic of the study cancer patients with cachexia stratified by TBIL.

\begin{tabular}{|c|c|c|c|}
\hline \multirow[t]{2}{*}{ Characteristic } & \multirow{2}{*}{$\begin{array}{l}\text { TBIL low } \\
\mathrm{n}=\mathbf{2 0 4 8}\end{array}$} & \multirow{2}{*}{$\begin{array}{l}\text { TBIL high } \\
\mathrm{n}=234\end{array}$} & \multirow[t]{2}{*}{$P$-value } \\
\hline & & & \\
\hline \multicolumn{4}{|l|}{ Population Characteristic } \\
\hline \multicolumn{4}{|l|}{ Sex (\%) } \\
\hline Male & $1153(56.3 \%)$ & $174(74.4 \%)$ & $<0.001$ \\
\hline Female & $895(43.7 \%)$ & $60(25.6 \%)$ & \\
\hline Age (mean (SD)) & $58.67(11.50)$ & $60.88(11.36)$ & 0.005 \\
\hline $\mathrm{BMI}, \mathrm{kg} / \mathrm{m}^{2}$, mean (SD) & $20.9(3.19)$ & $20.74(3.42)$ & 0.483 \\
\hline Diabetes, yes, n (\%) & $159(7.8 \%)$ & $26(11.1 \%)$ & 0.099 \\
\hline Hypertension, yes, n (\%) & $324(15.8 \%)$ & $48(20.5 \%)$ & 0.081 \\
\hline Hepatobiliary disease, yes, n (\%) & $162(7.9 \%)$ & $33(14.1 \%)$ & 0.002 \\
\hline Smoke, yes, n (\%) & $927(45.3 \%)$ & $121(51.7 \%)$ & 0.071 \\
\hline Alcohol, yes, n (\%) & $476(23.2 \%)$ & $65(27.8 \%)$ & 0.143 \\
\hline Tea, yes, n (\%) & $538(26.3 \%)$ & $63(26.9 \%)$ & 0.891 \\
\hline \multicolumn{4}{|l|}{ Clinical Characteristic } \\
\hline Lung cancer (\%) & $413(20.2 \%)$ & $34(14.5 \%)$ & 0.049 \\
\hline Liver cancer (\%) & $61(3.0 \%)$ & $35(15.0 \%)$ & $<0.001$ \\
\hline Gastric cancer (\%) & $458(22.4 \%)$ & $44(18.8 \%)$ & 0.245 \\
\hline Colorectal cancer (\%) & $502(24.5 \%)$ & $56(23.9 \%)$ & 0.908 \\
\hline Esophagus cancer (\%) & $184(9.0 \%)$ & $10(4.3 \%)$ & 0.02 \\
\hline Pancreatic cancer (\%) & $47(2.3 \%)$ & $21(9.0 \%)$ & $<0.001$ \\
\hline Gynecological and breast cancer (\%) & $256(12.5 \%)$ & $14(6.0 \%)$ & 0.005 \\
\hline Other cancer (\%) & $127(6.2 \%)$ & 20 (8.5\%) & 0.213 \\
\hline
\end{tabular}

Data are represented as mean (SD), median (interquartile range), or number (\%).

IQR, interquartile range; BMI, body mass index; KPS, Karnofsky Performance Status; TBIL, total bilirubin; DBIL, direct bilirubin; WBC, white blood cell; AST, alanine aminotransferase; ALT, aspartate aminotransferase; RBC, red blood cell count; MAC, mid-arm circumference; HGS, hand grip strength; PG-SGA, patient-generated subjective nutrition assessment.

For TBIL, low $<21.7 \mu \mathrm{mol} / \mathrm{L} ;$ high $\geq 21.7 \mu \mathrm{mol} / \mathrm{L}$ 


\begin{tabular}{|c|c|c|c|}
\hline \multirow[t]{2}{*}{ Characteristic } & TBIL low & TBIL high & \multirow[t]{2}{*}{$P$-value } \\
\hline & $n=2048$ & $\mathrm{n}=234$ & \\
\hline \multicolumn{4}{|l|}{ TNM stage (\%) } \\
\hline I & $176(8.6 \%)$ & $27(11.5 \%)$ & 0.083 \\
\hline II & $429(20.9 \%)$ & $40(17.1 \%)$ & \\
\hline III & $558(27.2 \%)$ & $53(22.6 \%)$ & \\
\hline IV & $885(43.2 \%)$ & $114(48.7 \%)$ & \\
\hline Radiotherapy, yes, n (\%) & $1178(57.5 \%)$ & $84(35.9 \%)$ & $<0.001$ \\
\hline Chemotherapy, yes, n (\%) & $977(47.7 \%)$ & $93(39.7 \%)$ & 0.025 \\
\hline TBIL, $\mu \mathrm{mol} / \mathrm{L}$, median (IQR),) & $10.96(4.07)$ & $60.06(70.76)$ & $<0.001$ \\
\hline DBIL, $\mu \mathrm{mol} / \mathrm{L}$, median (IQR), & $3.25(2.11)$ & $32.9(52.15)$ & $<0.001$ \\
\hline IBIL, $\mu \mathrm{mol} / \mathrm{L}$, median (IQR),) & $7.70(3.55)$ & $27.16(28.92)$ & $<0.001$ \\
\hline Albumin (mean (SD)) & $37.48(5.32)$ & $35.57(6.28)$ & $<0.001$ \\
\hline WBC, $10^{9} / \mathrm{L}$, mean $(\mathrm{SD})$ & $7.13(8.44)$ & $8.06(3.70)$ & 0.094 \\
\hline Neutrophil, $10^{9} / \mathrm{L}$, mean (SD) & $4.72(3.17)$ & $5.92(3.21)$ & $<0.001$ \\
\hline Lymphocyte, $10^{9} / \mathrm{L}$, mean (SD) & $1.5(0.71)$ & $1.38(1.13)$ & 0.031 \\
\hline AST (mean (SD)) & $25.57(20.89)$ & $85.57(271.77)$ & $<0.001$ \\
\hline ALT (mean (SD)) & $24.85(27.32)$ & $76.08(173.9)$ & $<0.001$ \\
\hline RBC (mean (SD)) & $4.07(0.65)$ & $3.96(0.78)$ & 0.013 \\
\hline Platelet (mean (SD)) & $246.5(100.39)$ & $212.59(104.7)$ & $<0.001$ \\
\hline KPS (mean (SD)) & $83.42(14.67)$ & 76.37 (20.32) & $<0.001$ \\
\hline MAC, cm, mean (SD) & $25.1(3.50)$ & $24.65(3.90)$ & 0.068 \\
\hline HGS, kg, mean (SD) & $23.34(9.90)$ & 22.57 (9.98) & 0.263 \\
\hline Anorexia, yes, n (\%) & $508(24.8 \%)$ & $90(38.5 \%)$ & $<0.001$ \\
\hline
\end{tabular}

Data are represented as mean (SD), median (interquartile range), or number (\%).

IQR, interquartile range; BMI, body mass index; KPS, Karnofsky Performance Status; TBIL, total bilirubin; DBIL, direct bilirubin; WBC, white blood cell; AST, alanine aminotransferase; ALT, aspartate aminotransferase; RBC, red blood cell count; MAC, mid-arm circumference; HGS, hand grip strength; PG-SGA, patient-generated subjective nutrition assessment.

For TBIL, low $<21.7 \mu \mathrm{mol} / \mathrm{L} ;$ high $\geq 21.7 \mu \mathrm{mol} / \mathrm{L}$ 


\begin{tabular}{|llll|}
\hline Characteristic & TBIL low & TBIL high & P-value \\
\hline $\mathbf{n = 2 0 4 8}$ & $\mathbf{n = 2 3 4}$ & \\
\hline Sarcopenia, yes, $\mathrm{n}(\%)$ & $1518(74.1 \%)$ & $165(70.5 \%)$ & 0.267 \\
\hline PG-SGA, mean (SD) & $9.31(4.44)$ & $11.53(4.73)$ & $<0.001$ \\
\hline EORTC QLQ-C30, mean (SD) & $52.06(10.85)$ & $58.3(13.68)$ & $<0.001$ \\
\hline Parenteral nutrition, yes, $\mathrm{n}(\%)$ & $335(16.4 \%)$ & $83(35.5 \%)$ & $<0.001$ \\
\hline Enteral nutrition, yes, $\mathrm{n}(\%)$ & $474(23.1 \%)$ & $90(38.5 \%)$ & $<0.001$ \\
\hline
\end{tabular}

Data are represented as mean (SD), median (interquartile range), or number (\%).

IQR, interquartile range; BMI, body mass index; KPS, Karnofsky Performance Status; TBIL, total bilirubin; DBIL, direct bilirubin; WBC, white blood cell; AST, alanine aminotransferase; ALT, aspartate aminotransferase; RBC, red blood cell count; MAC, mid-arm circumference; HGS, hand grip strength; PG-SGA, patient-generated subjective nutrition assessment.

For TBIL, low $<21.7 \mu \mathrm{mol} / \mathrm{L} ;$ high $\geq 21.7 \mu \mathrm{mol} / \mathrm{L}$

\subsection{Subgroup Analyses of Effect Modifiers of the Association of TBIL with Survival}

Stratified analyses were conducted to compare the relationship between TBIL and OS in several subgroups (Fig. 4 and Supplementary Table 3). Evidence of the association of a high TBIL level with an increased mortality risk was observed in the majority of subgroups. However, the similarassociations were not found among patients over 65 years old with abnormal BMI values, TNM stage III disease, lung cancer, radiotherapy, and abnormal neutrophil counts. Interestingly, interactions were observed between high TBIL levels and BMI, TNM stage, and tumor type (all $P$ for interaction $<0.1$ ). There were no other moderating effects of variables on the association between TBIL $(<27.1 \mu \mathrm{mol} / \mathrm{L}$ vs $\geq 27.1 \mu \mathrm{mol} / \mathrm{L})$ and all-cause mortality. Moreover, the differential effects of each variable were assessed (Supplementary Table 4). Three covariates, namely, low BMI, advanced tumor stage, and lung cancer, combined with elevated TBIL were associated with the worst prognosis in patients with cancer cachexia (Supplementary Fig. 4).

\subsection{Sensitivity analysis}

To test the robustness of our results, a sensitivity analysis was conducted by examining whether the association would change if individuals who died less than 6 months after the diagnosis of cancer cachexia or patients with hepatobiliary disease and hepatobiliary tumors were removed at baseline (Table 3). The results remained unchanged (adjusted $\mathrm{HR}, 1.44 ; 95 \% \mathrm{Cl}, 1.1-1.88$ for excluding patients dying within 6 months, adjusted HR, 1.52; $95 \% \mathrm{Cl}, 1.23-1.88$ for patients with hepatobiliary disease and hepatobiliary tumors). 
Table 3

Hazard risk for all-cause mortality in cachexia patients with low TBIL by excluding patients dying within 6 months or patients with hepatobiliary disease and hepatobiliary tumor burden

\begin{tabular}{|c|c|c|c|c|}
\hline TBIL & $\mathrm{HR} 95 \% \mathrm{Cl}$ & $P$-value & $\mathrm{HR} 95 \% \mathrm{Cl}$ & $P$-value \\
\hline Sensitive analysis & \multicolumn{2}{|c|}{ Excluding patients dying within 6 months } & \multicolumn{2}{|c|}{$\begin{array}{l}\text { Without hepatobiliary } \\
\text { disease and hepatobiliary } \\
\text { tumor burden }\end{array}$} \\
\hline As continuous (per SD) & $1.12(1.04,1.20)$ & 0.002 & $1.13(1.06,1.22)$ & $<0.001$ \\
\hline \multicolumn{5}{|l|}{ By TBIL ( $\mu \mathrm{mol} / \mathrm{L})$ cut-off } \\
\hline Low $(<21.7)$ & Ref. & & Ref. & \\
\hline High $(\geq 21.7)$ & $1.44(1.10,1.88)$ & 0.007 & $1.52(1.23,1.88)$ & $<0.001$ \\
\hline \multicolumn{5}{|l|}{ Tertile, $\mu \mathrm{mol} / \mathrm{L}$} \\
\hline Q1 (<9.1) & Ref. & & Ref. & \\
\hline Q2 (9.1-13.5) & $1.24(1.08,1.51)$ & 0.031 & $1.25(1.06,1.47)$ & 0.009 \\
\hline Q3 ( $\geq 15.2)$ & $1.32(1.08,1.62)$ & 0.008 & $1.42(1.20,1.68)$ & $<0.001$ \\
\hline \multicolumn{5}{|c|}{$\begin{array}{l}\text { Adjusted by gender, age, BMI, TNM stage, alcohol consumption, tumor types, TNM stage, } \\
\text { radiotherapy, chemotherapy, KPS, albumin, RBC, platelet, HGS, sarcopenia, neutrophils, AST, ALT, } \\
\text { EORTC QLQ-C30 score. }\end{array}$} \\
\hline
\end{tabular}

\section{Discussion}

To our knowledge, this study is the largest to directly evaluate the association of serum TBIL with allcause mortality in patients with cachexia. The distribution of TBIL concentration was associated with several types of tumors in this cohort. Furthermore, TBIL was an independent prognostic marker in multivariate analysis. We then calculated the TBIL cutoff point for the prediction of mortality in Chinese cancer patients with cachexia. Using this cutoff point, we found that a high TBIL level was strongly associated with all-cause mortality. Further analysis showed that the mortality risk was especially elevated in patients with high TBIL levels who had low BMI values, advanced cancer stages, and digestive system cancers. In the sensitivity analysis, the exclusion of patients with evidence of hepatobiliary disease and hepatobiliary tumors did not change the positive association of TBIL with the risk of allcause mortality. In general, serum TBIL can serve as an indicator for the daily assessment of the prognosis of patients with cancer cachexia.

Cancer cachexia is a multi-organ syndrome that involves more than just skeletal muscle pathogenesis 7,16 . Hepatic function can directly or indirectly cause whole-body energy expenditure and increased mortality of patients with cancer cachexia ${ }^{17,18}$. BLB (TBIL, DBIL, and IBIL), albumin, ALT, and AST are frequently used as indicators of liver function. Elevated physiological marker levels reflect liver 
disease and hepatocyte injury. Similar to previous studies ${ }^{19-21}$, these liver parameters, except DBIL and IBIL, were independent prognostic factors in this population-based cohort study. However, there was no association between serum TBIL and all-cause mortality in the subgroup of patients with hepatocellular carcinoma (HCC) and cachexia ( $\mathrm{HR}, 1.40 ; 95 \% \mathrm{Cl}, 0.68-2.87, P=0.641$, data not shown). It is conceivable that this is attributable to the complexity of pathological changes in the liver, and other mechanisms should be investigated. For example, the different effects of the various forms (alpha- to delta-) of serum TBIL should be explored. Another explanation might be the limited number of HCC participants in our cohort.

To date, a few studies have investigated the associations of BLB with other cancer risks. Moderately elevated serum BLB levels were found to be associated with a markedly reduced prevalence of CRC ${ }^{22}$. Meanwhile, two previous studies reported thatserum TBIL was inversely correlated with lung cancer mortality. One study proposed that relatively higher levels of BLB may protect people against lung cancers $^{23}$. Laura et al also identified an association between elevated levels of serum BLB due to genetic factors and lower rates of lung cancer ${ }^{24}$. Reports concerning breast cancer are consistent with these findings ${ }^{25,26}$. Unfortunately, several studies have reported conflicting results regarding the relationship between the BLB concentration and cancer. Two cohort studies confirmed the absence of an association between $B L B$ and $C R C^{27,28}$. This may be due to the fact that $B L B$ has antioxidant and antineoplastic effects $^{29}$, and a number of studies have demonstrated that BLB inhibits the activity of cytotoxic $T$ lymphocytes and promotes $T$ regulatory cell $\left(T_{\text {reg }}\right)$ expansion ${ }^{30,31}$. Overall, consistent evidence that plasma BLB has a protective effect against cancer is lacking. In our study, in patients with digestive system tumors, there was a significant negative correlation between the TBIL level and overall mortality. We conjecture that BLB signaling to the gut microbiota and its regulation of the liver-gut axis may have led to the above results. This emphasizes the importance of further research to elucidate the current results and confirm whether TBIL can be used to predict survival in patients with cachexia. Further investigation of the genetic factors affecting BLB is also warranted. UDP-glucuronosyltransferase (UGT1A1) is a key enzyme involved in the metabolism of BLB, and its mutations cause BLB metabolism disorders, such as Gilbert's syndrome ${ }^{32,33}$. The clinical impacts of UGT1A1 promoter gene variation have been thoroughly studied in CRC patients ${ }^{34}$. UGT1A1*28 allele carrier status was found to be inversely associated with the development of CRC in males. Nevertheless, the limitation in most analyses was the lack of BLB measurements. In the future study, we will focus on combining genetic analyses with BLB analyses to further elucidate the mechanism.

Patients who are malnourished have a poor response to antitumor therapy and an elevated risk of cachexia-related mortality ${ }^{35}$.Our interaction analyses established an association between TBIL and BMI, which is a nutrition status indicator. In the subgroup analysis, TBIL was an effective indicator of prognosis in patients with normal BMI values. Patients with high TBIL levels and low BMI values had shorter survival durations, according to the KM analysis. The mechanisms underlying this association are unclear. Interestingly, patients with high BMI tended to have a better prognosis, indicating that obesity 
might have a protective role against cancer cachexia. Consistent with our study, several meta-analyses reported that women with higher BMI values had a lower lung cancer risk and better prognosis ${ }^{36,37}$.

Some limitations of our study need to be considered. Serum TBIL concentrations change dynamically in healthy people, especially depending on the fasting state. The changes in TBIL in patients with cancer cachexia should be taken into consideration. Follow-up studies are warranted to detect the impacts of dynamic changes in TBIL on mortality in patients with cancer cachexia. Another limitation is that some unmeasured indicators, such as alkaline phosphatase (ALP) and lactate dehydrogenase (LDH), which also reflect liver function, or other measured confounding factors may have affected our results.

\section{Conclusion}

In conclusion, we established for the first time the cutoff point of TBIL for the prediction of mortality in patients with cancer cachexia. A lower level of serum TBIL was associated with a decreased risk of allcause mortality. This is important, as serum TBIL is an easily assessable, highly reproducible, and inexpensive biomarker and might be a promising clinical tool to find patients with cancer cachexia at risk of mortality. Long-term studies on the development and progression of cancer cachexia are needed to evaluate the causal associations between serum TBIL levels and cancer cachexia. A comprehensive understanding of these mechanisms may guide the selection of appropriate clinical treatments.

\section{Abbreviations}

AST

aspartate transaminase

ALT

alanine transaminase

$\mathrm{BMI}$

body mass index

$\mathrm{Cl}$

confidence interval

DBIL

direct bilirubin

EORTC QLQ-C30

European Organization for Research and Treatment of Cancer QLQ-C30

GLIM

Global Leadership Initiative on Malnutrition

HGS

hand grip strength

HR

hazard ratio

IBIL 
indirect bilirubin

MAC

mid-arm circumference

OS

overall survival

PG-SGA

patient-generated subjective nutrition assessment

RBC

red blood cell

SMI

skeletal muscle index

TBIL

total bilirubin

WBC

white blood cell

\section{Declarations}

\section{Ethics approval and consent to participate}

The authors are accountable for all aspects of the work in ensuring that questions related to the accuracy or integrity of any part of the work are appropriately investigated and resolved. Prior to data collection, all study participants signed an informed consent form This study was approved by the institutional review board of Beijing Shijitan Hospital (ChiCTR1800020329). The study was conducted in accordance with the Declaration of Helsinki (as revised in 2013). The study was approved by institutional review board of Beijing Shijitan Hospital (ChiCTR1800020329) and individual consent for this retrospective analysis was waived. The authors acquired written informed consent from the patient.

\section{Consent for publication}

Consent for publication was obtained from all co-author.

\section{Availability of supporting data}

Data will be made available after request application and approval.

\section{Competing interests}

The authors have no conflicts of interest to declare.

\section{Funding}


This work was supported by the National Key Research and Development Program to Dr. Hanping Shi (No. 2017YFC1309200)

\section{Authors' contributions}

(I) Conception and design: Han-Ping Shi; (II) Administrative support: Han-Ping Shi; (III) Provision of study material or patients: All authors; (IV) Collection and assembly of data: Qi Zhang, Xi Zhang, Guo-Tian Ruan; (V) Data analysis and interpretation: Xiang-Rui Li,MD, Kang-Ping Zhang; (VI) Manuscript writing: All authors; (VII) Final approval of manuscript: All authors

\section{Acknowledgments}

The authors thank all members of Investigation on Nutrition Status and Its Clinical Outcome of Common Cancers (INSCOC) for their assistance in data collection and follow-up.

\section{References}

1. Fearon K, Strasser F, Anker SD, et al. Definition and classification of cancer cachexia: an international consensus. Lancet Oncol. 2011;12(5):489-495.

2. Baracos VE, Martin L, Korc M, Guttridge DC, Fearon KCH. Cancer-associated cachexia. Nat Rev Dis Primers. 2018;4:17105.

3. Baracos VE. Pitfalls in defining and quantifying cachexia. J Cachexia Sarcopenia Muscle. 2011;2(2):71-73.

4. Yavuzsen T, Davis MP, Walsh D, LeGrand S, Lagman R. Systematic review of the treatment of cancerassociated anorexia and weight loss. J Clin Oncol. 2005;23(33):8500-8511.

5. Blum D, Stene GB, Solheim TS, et al. Validation of the Consensus-Definition for Cancer Cachexia and evaluation of a classification model-a study based on data from an international multicentre project (EPCRC-CSA). Ann Oncol. 2014;25(8):1635-1642.

6. Fearon KC, Glass DJ, Guttridge DC. Cancer cachexia: mediators, signaling, and metabolic pathways. Cell Metab. 2012;16(2):153-166.

7. Schmidt SF, Rohm M, Herzig S, Berriel Diaz M. Cancer Cachexia: More Than Skeletal Muscle Wasting. Trends Cancer. 2018;4(12):849-860.

8. Martignoni ME, Dimitriu C, Bachmann J, et al. Liver macrophages contribute to pancreatic cancerrelated cachexia. Oncol Rep. 2009;21(2):363-369.

9. Friesen DE, Baracos VE, Tuszynski JA. Modeling the energetic cost of cancer as a result of altered energy metabolism: implications for cachexia. Theor Biol Med Model. 2015;12:17.

10. Vitek L. The role of bilirubin in diabetes, metabolic syndrome, and cardiovascular diseases. Front Pharmacol. 2012;3:55.

11. Temme EH, Zhang J, Schouten EG, Kesteloot H. Serum bilirubin and 10-year mortality risk in a Belgian population. Cancer Causes Control. 2001;12(10):887-894. 
12. Perlstein TS, Pande RL, Creager MA, Weuve J, Beckman JA. Serum total bilirubin level, prevalent stroke, and stroke outcomes: NHANES 1999-2004. Am J Med. 2008;121(9):781-788 e781.

13. Novotny L, Vitek L. Inverse relationship between serum bilirubin and atherosclerosis in men: a metaanalysis of published studies. Exp Biol Med (Maywood). 2003;228(5):568-571.

14. Valentova $M$, von Haehling $S$, Krause $C$, et al. Cardiac cachexia is associated with right ventricular failure and liver dysfunction. Int J Cardiol. 2013;169(3):219-224.

15. Dell'Aquila E, Cremolini C, Zeppola T, et al. Prognostic and predictive role of neutrophil/lymphocytes ratio in metastatic colorectal cancer: a retrospective analysis of the TRIBE study by GONO. Ann Oncol. 2018;29(4):924-930.

16. Anker MS, Holcomb R, Muscaritoli M, et al. Orphan disease status of cancer cachexia in the USA and in the European Union: a systematic review. J Cachexia Sarcopenia Muscle. 2019;10(1):22-34.

17. Argiles JM, Stemmler B, Lopez-Soriano FJ, Busquets S. Inter-tissue communication in cancer cachexia. Nat Rev Endocrinol. 2018;15(1):9-20.

18. Rosa-Caldwell ME, Brown JL, Lee DE, et al. Hepatic alterations during the development and progression of cancer cachexia. Appl Physiol Nutr Metab. 2020;45(5):500-512.

19. Yeun JY, Kaysen GA. Factors influencing serum albumin in dialysis patients. Am J Kidney Dis. 1998;32(6 Suppl 4):S118-125.

20. Sharma R, Hook J, Kumar M, Gabra H. Evaluation of an inflammation-based prognostic score in patients with advanced ovarian cancer. Eur J Cancer. 2008;44(2):251-256.

21. Liu X, Meng QH, Ye Y, Hildebrandt MA, Gu J, Wu X. Prognostic significance of pretreatment serum levels of albumin, LDH and total bilirubin in patients with non-metastatic breast cancer. Carcinogenesis. 2015;36(2):243-248.

22. Zucker SD, Horn PS, Sherman KE. Serum bilirubin levels in the U.S. population: gender effect and inverse correlation with colorectal cancer. Hepatology. 2004;40(4):827-835.

23. Horsfall LJ, Rait G, Walters K, et al. Serum bilirubin and risk of respiratory disease and death. JAMA. 2011;305(7):691-697.

24. Horsfall LJ, Burgess S, Hall I, Nazareth I. Genetically raised serum bilirubin levels and lung cancer: a cohort study and Mendelian randomisation using UK Biobank. Thorax. 2020;75(11):955-964.

25. Alexandre J, Bleuzen P, Bonneterre J, et al. Factors predicting for efficacy and safety of docetaxel in a compassionate-use cohort of 825 heavily pretreated advanced breast cancer patients. J Clin Oncol. 2000;18(3):562-573.

26. Ching S, Ingram D, Hahnel R, Beilby J, Rossi E. Serum levels of micronutrients, antioxidants and total antioxidant status predict risk of breast cancer in a case control study. J Nutr. 2002;132(2):303-306.

27. Ko WF, Helzlsouer KJ, Comstock GW. Serum albumin, bilirubin, and uric acid and the anatomic sitespecific incidence of colon cancer. J Natl Cancer Inst. 1994;86(24):1874-1875.

28. Ioannou GN, Liou IW, Weiss NS. Serum bilirubin and colorectal cancer risk: a population-based cohort study. Aliment Pharmacol Ther. 2006;23(11):1637-1642. 
29. Stocker R, Yamamoto Y, McDonagh AF, Glazer AN, Ames BN. Bilirubin is an antioxidant of possible physiological importance. Science. 1987;235(4792):1043-1046.

30. Haga Y, Tempero MA, Zetterman RK. Unconjugated bilirubin inhibits in vitro cytotoxic T lymphocyte activity of human lymphocytes. Biochim Biophys Acta. 1996;1317(1):65-70.

31. Rocuts $F$, Zhang $X$, Yan J, et al. Bilirubin promotes de novo generation of T regulatory cells. Cell Transplant. 2010;19(4):443-451.

32. Erlinger $S$, Arias IM, Dhumeaux D. Inherited disorders of bilirubin transport and conjugation: new insights into molecular mechanisms and consequences. Gastroenterology. 2014;146(7):1625-1638.

33. Claridge LC, Armstrong MJ, Booth C, Gill PS. Gilbert's syndrome. BMJ. 2011;342:d2293.

34. Jiraskova A, Novotny J, Novotny L, et al. Association of serum bilirubin and promoter variations in HMOX1 and UGT1A1 genes with sporadic colorectal cancer. Int J Cancer. 2012;131(7):1549-1555.

35. Pressoir M, Desne S, Berchery D, et al. Prevalence, risk factors and clinical implications of malnutrition in French Comprehensive Cancer Centres. Br J Cancer. 2010;102(6):966-971.

36. Shen N, Fu P, Cui B, Bu CY, Bi JW. Associations between body mass index and the risk of mortality from lung cancer: A dose-response PRISMA-compliant meta-analysis of prospective cohort studies. Medicine (Baltimore). 2017;96(34):e7721.

37. Yang Y, Dong J, Sun K, et al. Obesity and incidence of lung cancer: a meta-analysis. Int J Cancer. 2013;132(5):1162-1169.

\section{Figures}




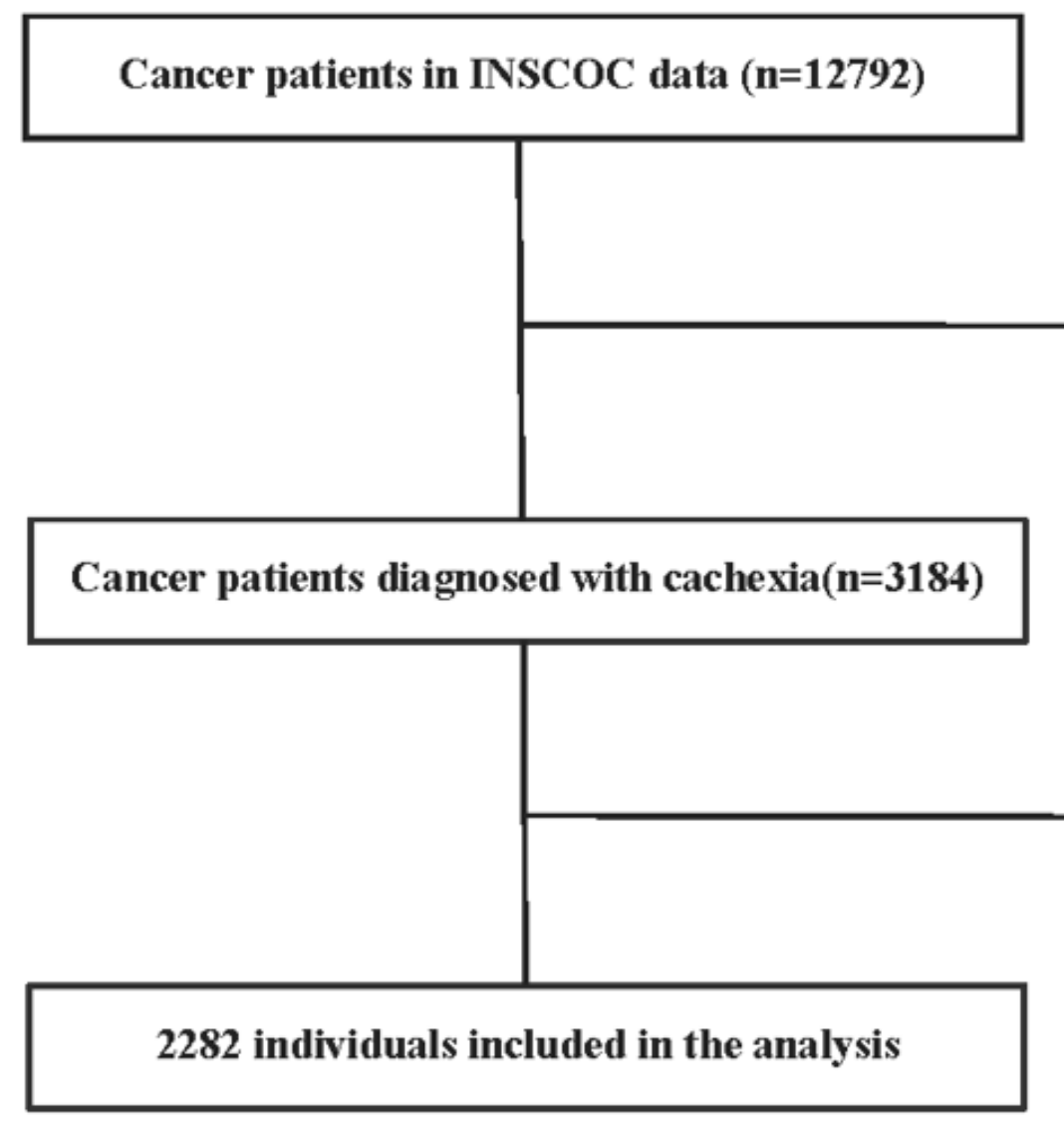

Screening of patients with cachexia

by 2011 international consensus of cachexia

\section{Excuded n=902}

383: fasting blood sample

453: EORTC QLQ-C30 score

38: Body mass index

28: follow up data

Figure 1

Diagram of participants included in the analysis 


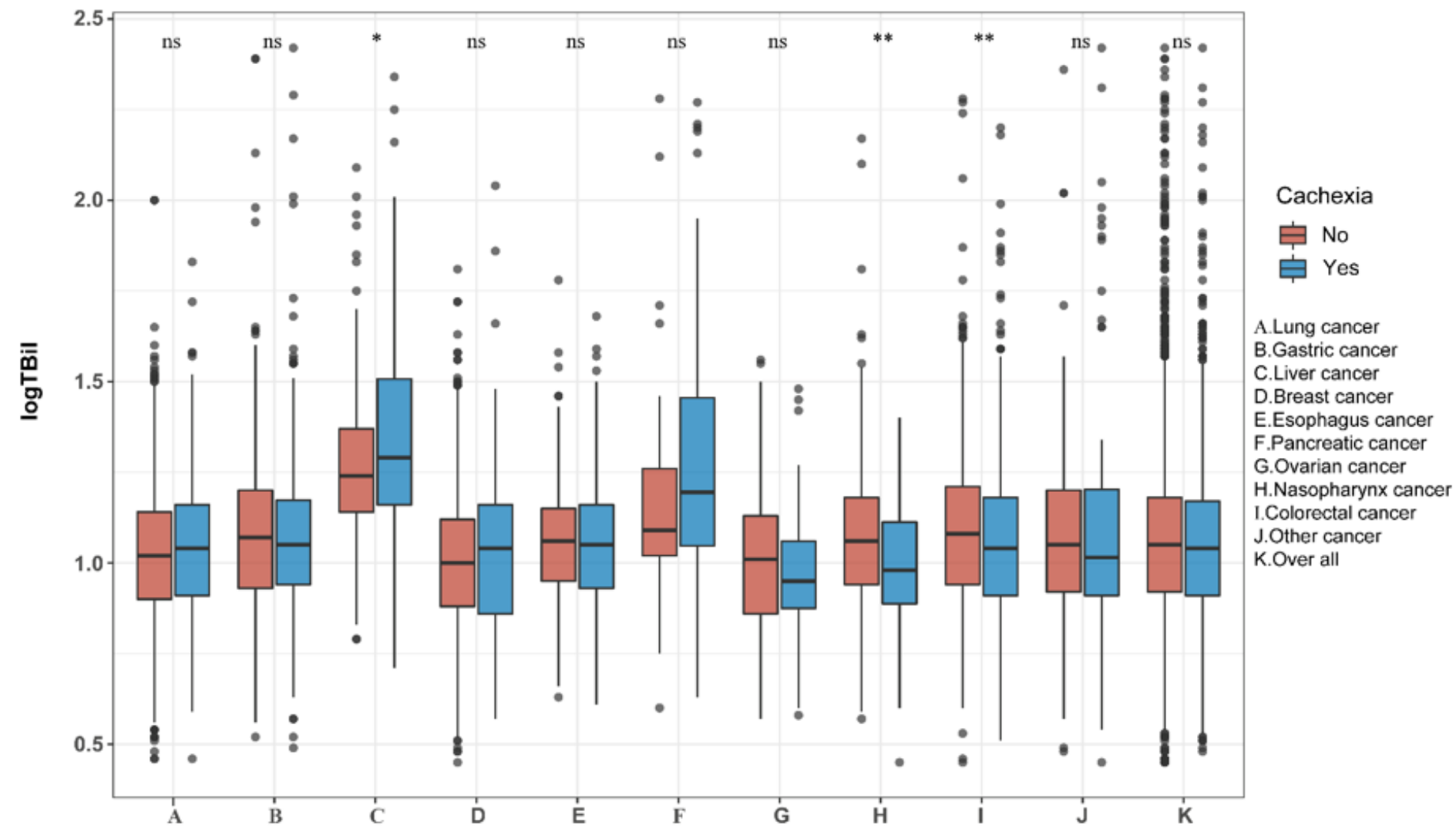

ns $P$-value $>0.05$, * $P$-value $<0.05$, ** $P$-value $<0.01$.

Figure 2

TBIL (log transformation) in different cancer types stratified by whether patients had cachexia 


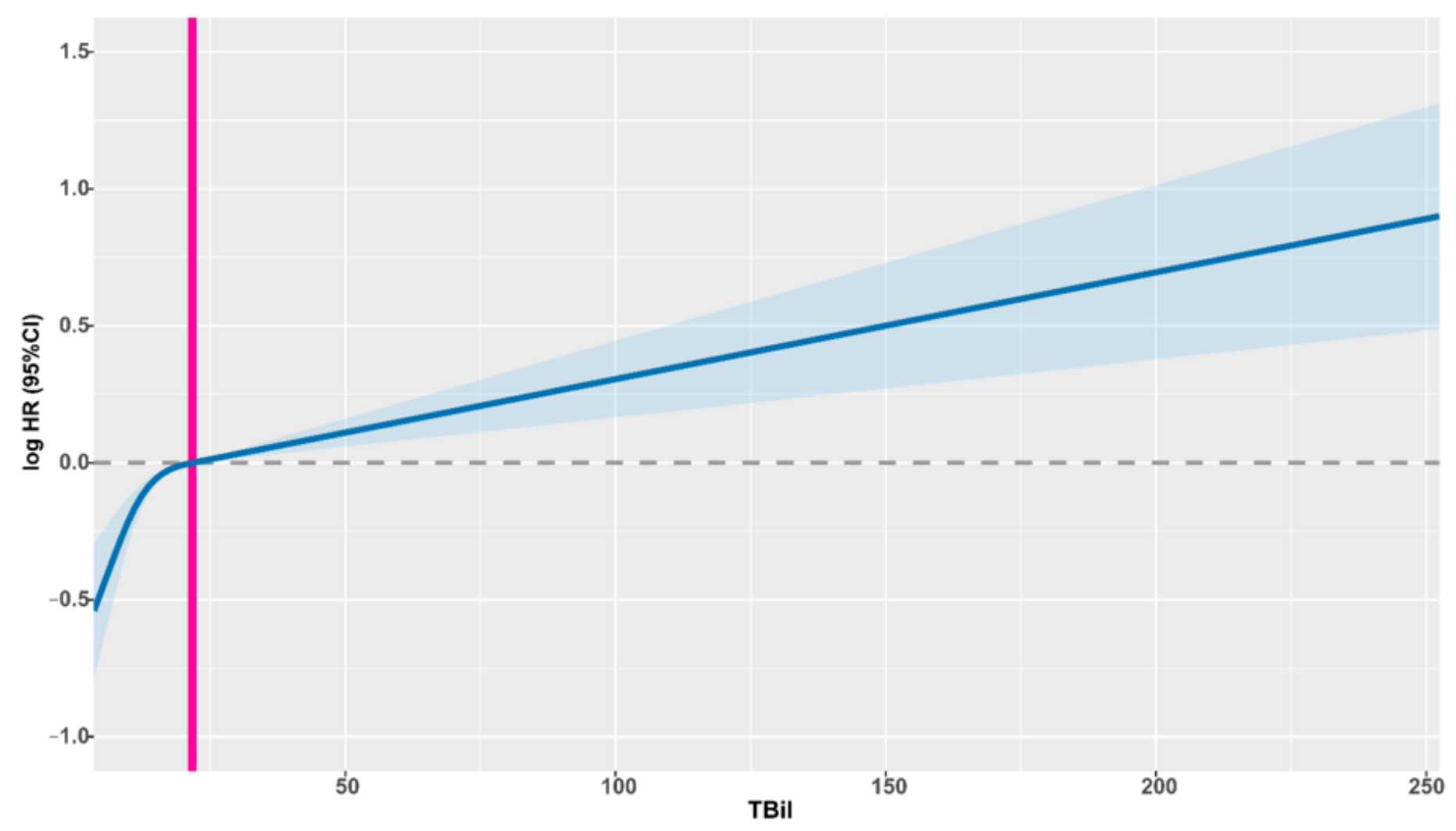

The spline was adjusted by sex, age, BMI, tumor type, TNM stage, radiotherapy, chemotherapy, KPS, albumin, TBIL, RBC count, platelet count, sarcopenia, HGS, anorexia, and EORTC QLQ-C30 score.

\section{Figure 3}

Association between TBIL (continuous) and overall survival. 


\begin{tabular}{|c|c|c|c|c|}
\hline $\begin{array}{c}\text { Characteristic } \\
\text { Gender }\end{array}$ & HR 95\%Cl & & $P$ value & $\begin{array}{c}P \text { for interaction } \\
0.516\end{array}$ \\
\hline Male & $1.68(1.11,2.54)$ & $\longmapsto$ & 0.010 & \\
\hline Female & $1.61(1.30,1.99)$ & $\mapsto-1$ & $<0.001$ & \\
\hline Age, year & & & & 0.659 \\
\hline$<65$ & $1.83(1.44,2.31)$ & •— & $<0.001$ & \\
\hline$\geqslant 65$ & $1.27(0.91,1.78)$ & $\mapsto$ & 0.160 & \\
\hline BMI, $\mathrm{kg} / \mathrm{m}^{2}$ & & & & 0.055 \\
\hline$<18.5$ & $1.31(0.92,1.86)$ & $\mapsto$ & 0.130 & \\
\hline $18.5-24$ & $1.90(1.49,2.44)$ & $\longmapsto$ & $<0.001$ & \\
\hline$>24$ & $1.69(0.86,3.34)$ & 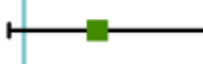 & 0.130 & \\
\hline TNM stage & & & & 0.096 \\
\hline I & $8.45(2.78,25.64)$ & & $<0.001$ & \\
\hline II & $2.33(1.24,4.37)$ & $\longrightarrow$ & 0.010 & \\
\hline III & $1.46(0.94,2.28)$ & $\longmapsto$ & 0.090 & \\
\hline IV & $1.57(1.25,1.97)$ & •ー & $<0.001$ & \\
\hline Tumor type & & & & 0.089 \\
\hline Lung & $1.15(0.73,1.80)$ & $-\longrightarrow$ & 0.550 & \\
\hline Digestive & $1.86(1.50,2.30)$ & $\mapsto$ & $<0.001$ & \\
\hline Other & $2.44(1.02,5.84)$ & & 0.050 & \\
\hline KPS & & & & 0.203 \\
\hline$>70$ & $1.70(1.37,2.11)$ & $\mapsto$ & $<0.001$ & \\
\hline$\leqslant 70$ & $1.63(1.12,2.35)$ & $\longmapsto$ & 0.010 & \\
\hline Albumin,g/L & & & & 0.821 \\
\hline Normal & $1.50(1.13,2.00)$ & $\mapsto$ & 0.010 & \\
\hline Abnormal & $1.61(1.24,2.07)$ & $\mapsto$ & $<0.001$ & \\
\hline HGS,kg & & & & 0.299 \\
\hline Normal & $1.74(1.28,2.37)$ & $\longmapsto$ & $<0.001$ & \\
\hline Abnormal & $1.65(1.30,2.10)$ & $\mapsto \bullet$ & $<0.001$ & \\
\hline
\end{tabular}

The model was adjusted by sex, age, BMI, TNM stage, tumor type, TNM stage, radiotherapy, chemotherapy, KPS, albumin, RBC count, platelet count, HGS, sarcopenia, neutrophil count, and EORTC QLQ-C30 score, as appropriate.

Figure 4

Associations between TBIL stratified by the cutoff point as low $(<21.7 \mu \mathrm{mol} / \mathrm{L})$ and high $(\geq 21.7 \mu \mathrm{mol} / \mathrm{L})$ and overall survival in various subgroups 
This is a list of supplementary files associated with this preprint. Click to download.

- SupplementaryMaterial.docx 